\title{
Magnetic resonance imaging metal artifact reduction for eye plaque patient with dental braces
}

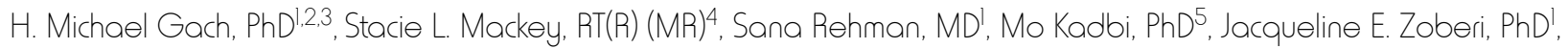 \\ Jose Garcia-Ramirez, MS', Perry W. Grigsby, MD' \\ 'Department of Radiation Oncology, ${ }^{2}$ Department of Radiology, ${ }^{3}$ Department of Biomedical Engineering, Washington University in St. Lovis, \\ St. Lovis, MO. ${ }^{4}$ Department of Radiation Oncology, Barnes Jewish Hospital, St. Lovis, MO, ${ }^{5}$ Philips Healthcare, Cleveland, OH, USA
}

\begin{abstract}
Purpose: To determine if metal artifact reduction can minimize magnetic susceptibility artifacts in the orbits for an eye plaque brachytherapy patient with metallic dental braces.

Material and methods: A 62-year-old female patient with a choroidal melanoma in the right eye received a $1.5 \mathrm{~T}$ magnetic resonance imaging (MRI) simulation for 3D eye plaque brachytherapy planning. The protocol included conventional 3D $\mathrm{T}_{1}$-weighted and 2D $\mathrm{T}_{2}$-weighted MRIs. A vendor-supplied $\mathrm{T}_{2}$-weighted metal artifact reduction sequence was added to the protocol to reduce magnetic susceptibility artifacts from the metallic dental braces. The metal artifact reduction sequence combined turbo spin echo acquisitions, high RF excitation and readout bandwidths, and view angle tilting and slice encoding for metal artifact correction with z-shimming to correct in-plane and through-plane image distortions, respectively.

Results: Dental braces caused significant signal loss and image distortion in the orbits on the conventional $\mathrm{T}_{1}$-weighted and $\mathrm{T}_{2}$-weighted MRIs, and the MRIs were unusable for treatment planning. The metal artifact reduction sequence with $13 \mathrm{z}$-phase encodes minimized distortion and signal loss in the orbits, allowing the tumor to be clearly delineated.

Conclusions: $\mathrm{T}_{2}$-weighted MRI with metal artifact reduction was successfully applied to minimize artifacts in the orbits resulting from the dental braces, thus allowing the MRIs to be used in 3D brachytherapy treatment planning.

J Contemp Brachytherapy 2017; 9, 5: 490-495

DOI: https://doi.org/10.5114/jcb.2017.71184
\end{abstract}

Key words: dental braces, eye plaque, metal artifact reduction, orbits, SEMAC.

\section{Purpose}

Episcleral eye plaque brachytherapy is an effective treatment for ocular melanomas that avoids removal of the affected eye [1,2]. The treatment involves suturing a radioactive plaque to the sclera adjacent to the tumor. The plaque remains in place until the desired dose is administered. Radionuclides may include gamma emitters ${ }^{125} \mathrm{I},{ }^{103} \mathrm{Pd},{ }^{192} \mathrm{Ir}$, and beta emitters ${ }^{106} \mathrm{Ru}$ and ${ }^{90} \mathrm{Sr}[3,4,5,6]$.

Fundoscopy and ultrasonography are the current imaging standards for assessing the dimensions of intraocular melanomas for eye plaque brachytherapy [7]. 3D treatment planning based on computed tomography (CT) was demonstrated for episcleral plaque brachytherapy, but tumor delineation was unsatisfactory due to poor soft tissue contrast [8].

At our institution, eye plaque patients typically receive fundoscopy, ultrasound, and magnetic resonance imaging (MRI) for brachytherapy treatment planning [9]. MRI has superior soft tissue contrast to CT and is nonionizing. $\mathrm{T}_{1}$-weighted (T1W) and $\mathrm{T}_{2}$-weighted (T2W) (MRI) were shown to provide excellent spatial delineation of the tumor $[10,11,12]$. The T1W MRIs have excellent contrast between the tumor (bright) and the vitreous humor (dark), but cannot distinguish between retinal detachment (if present) and the tumor. On T2W MRI, the tumor appears hypointense compared to the vitreous humor and retinal detachment (if present).

Orthodontic appliances typically contain stainless steel and titanium that may produce severe magnetic susceptibility artifacts in the head and neck, and degrade the diagnostic quality of the MRI exam [13,14]. Temporary removal of orthodontic devices is frequently justified to minimize susceptibility artifacts when diagnostic MRIs are required [15]. Obviously, it is far preferable to leave the orthodontic devices intact if the MRI can be successfully performed with minimal artifacts. This case report describes the use of a metal artifact reduction MRI pulse sequence to reduce magnetic susceptibility artifacts related to dental braces in an eye plaque brachytherapy patient.
Address for correspondence: H. Michael Gach, PhD, Washington University in St. Louis, St. Louis, MO, 4921 Parkview Place, Campus Box 8224, St. Louis, MO 63110, USA, phone: +1 314 286-1645,

fax: +1 314 362-8521, « e-mail: gachhm@wustl.edu
Received: 16.12 .2016

Accepted: 12.10 .2017

Published: 30.10.2017 


\section{Material and methods}

A 62-year-old female patient presented with a slowly growing eye tumor consistent with a right choroidal melanoma. She was scheduled to receive definitive therapy with eye plaque brachytherapy and received an MRI simulation for treatment planning. The MRI was performed using a Philips Ingenia 1.5 Tesla MRI scanner (Philips Healthcare, The Netherlands). The patient was placed supine in a 15 channel phased array head coil. At isocenter, the patient was instructed to stare at a decal that is located on the top of the bore and to minimize blinking.

The nominal MRI protocol for our eye plaque patients includes the following pulse sequences: 1) three-plane T1W scouts; 2) 2D T2W turbo spin echo (TSE) MRI; and 3) 3D T1W MRI. The pulse sequence parameters are summarized in Table 1 . The structural sequences are acquired in the axial orientation with the phase encode in the right/left direction to prevent flow artifacts from appearing in the orbits. Intravenous MRI contrast is not used in these studies.

MRI safety screening identified that the patient had dental braces with metal and was claustrophobic. Dental braces are generally considered safe for nominal MRI field strengths. However, we limit the specific absorption rate to $<2.5 \mathrm{~W} / \mathrm{kg}$ to minimize the risk of radiofrequency heating. The patient was coached to immediately use the squeeze bulb to alert the technologist in case of experiencing unusual heating or other adverse events.

The braces produced magnetic susceptibility artifacts observed as dramatic signal loss in the anterior portion of the head on the T1W scout images and image geometric distortion in the orbit area on T2W. The patient was withdrawn from the bore with the intention to abort the exam. The MRI technologist contacted the MRI physicist and physician to determine the best course of action.

In an effort to salvage the exam and obtain satisfactory MRI planning information for brachytherapy, the team decided to rescan the patient using Philips' multislice 2D orthopedic metal artifact reduction (O-MAR) research sequence. The O-MAR sequence combines TSE acquisitions, high RF excitation, and readout bandwidths as well as view angle tilting (VAT) and slice encoding for metal artifact correction (SEMAC) with z-shimming to correct in-plane and through-plane image distortions, respectively $[16,17]$. The SEMAC strength can be adjusted as weak, medium, and strong corresponding to specific frequency ranges per slice (z-phase encodes) of $\pm 5 \mathrm{kHz}(7), \pm 10 \mathrm{kHz}$ (13), and $\pm 15 \mathrm{kHz}$ (21), respectively. The acquisition time increases with the number of z-phase encodes, so it is advisable to use the weakest setting that corrects the artifact. We routinely use the weak SEMAC setting for cervical cancer patients with titanium tandems that would otherwise produce susceptibility artifacts with millimeter dimensions using conventional sequences [18]. The O-MAR sequence can currently be run with 4 contrast options: 1) $\mathrm{T} 1 \mathrm{~W} ; 2) \mathrm{T} 2 \mathrm{~W}$; 3) proton density weighting (PDW); and 4) short TI inversion recovery (STIR).

The Philips O-MAR product sequence (version 5.2 and later) is FDA approved. For this study, our Philips MRI used an earlier software version (version 5.1.7), and the O-MAR pulse sequence was run as a software patch. Our Institutional Review Board allowed us to use the O-MAR research sequence for clinical use without requiring informed consent, due to the clinical benefit/risk ratio and the similarity between the research and product sequences.

The O-MAR software patch was installed by running a program that updates the pulse sequence. The patient was returned to isocenter, and an additional set of scouts and conventional 3D T1W images were acquired. We also acquired T2W O-MAR MRIs (Table 1). We selected the medium SEMAC setting (13 z-phase encodes) due to the severity of the artifact and in the interest of time. The patient was claustrophobic and wanted to end the exam, therefore no further sequences (e.g., T1W with O-MAR) or pulse sequence parameter options (e.g., increasing the receiver bandwidth) were completed.

The MRIs were imported into an Eclipse Brachyvision treatment planning system (Varian Medical Systems, Palo Alto, CA, USA), and used to construct a 3D treatment plan as briefly described herein. The details of our MRI-based eye plaque brachytherapy methodology were recently published [9].

Table 1. Pulse sequence parameters

\begin{tabular}{lcccc}
\hline Parameter & T1W Scout & 3D T1W & 2D T2W & 2D T2W with SEMAC* \\
\hline TE/TR (ms) & $5 / 15$ & $5 / 25$ & $120 / 7924$ & $100 / 4099$ \\
\hline No. of slices & 5 & 42 & 33 & 41 \\
\hline Slice thickness/gap (mm) & $10 / 10$ & $1.2 / 0$ & $1.5 / 0.2$ & $2 / 0$ \\
\hline Flip angle & $20^{\circ}$ & $30^{\circ}$ & $90^{\circ}$ & $90^{\circ}$ \\
\hline $\begin{array}{l}\text { Acquired and reconstructed } \\
\text { voxel sizes (mm) }\end{array}$ & $2.38 \times 1.00$ & $1.22 \times 1.18$ & $1.17 \times 0.98$ & $1.22 \times 0.99$ \\
\hline Receiver BW (Hz/Pixel) & $0.94 \times 0.94$ & $0.94 \times 0.94$ & 139 & $0.97 \times 0.97$ \\
\hline $\begin{array}{l}\text { No. of averages } \\
\text { Acquisition time (s) }\end{array}$ & 187 & 109 & 3 & 1 \\
\hline $\begin{array}{l}\text { *SEMAC used 13 z-phase encodes } \\
\text { SEMAC - slice encoding for metal artifact correction }\end{array}$ & 18 & 1 & 293 & 406 \\
\hline
\end{tabular}


The tumor and critical structures (e.g., lens, macula, and optic nerve) were contoured. The treatment plan was designed to provide one fraction of $85 \mathrm{~Gy}$ delivered over 96 hours from Collaborative Ocular Melanoma Study (COMS) eye plaques loaded with ${ }^{125}$ I seeds. The plaque dosimetry used a TG-43 based homogenous dose calculation [19]. The seed groups were based on TG-129 seed center coordinates [20].

\section{Results}

The T1W MRIs without metal artifact reduction are shown in Figure 1. There is severe signal degradation in the orbits. The T2W MRIs with and without SEMAC are shown in Figure 2. O-MAR minimized the susceptibility artifact in the region of the tumor for the T2W MRIs (Figure 2). The dimensions of the eyes were $20-30 \%$ longer in the anterior/posterior (readout) direction for the $\mathrm{T} 2 \mathrm{~W}$ images without O-MAR versus with O-MAR. The specific absorption rate for the T2W sequences with and without O-MAR were 0.3 and $0.2 \mathrm{~W} / \mathrm{kg}$, respectively. The patient experienced no unusual heating during the MRI exam.

The MRIs with O-MAR were used to create a 3D model (Figure 3 and Table 2), which was necessary due to the irregular shape of the target volume and its proximity to critical structures (e.g., lens, optic disk, and fovea). On fundoscopy, the tumor was measured to be $7.9 \times 8.7 \mathrm{~mm}$ with an apical height of $3.34 \mathrm{~mm}$. On ultrasound, the tumor was measured to be $7.96 \times 8.72 \mathrm{~mm}$ with an apical height of $3.29 \mathrm{~mm}$. The tumor dimension was measured
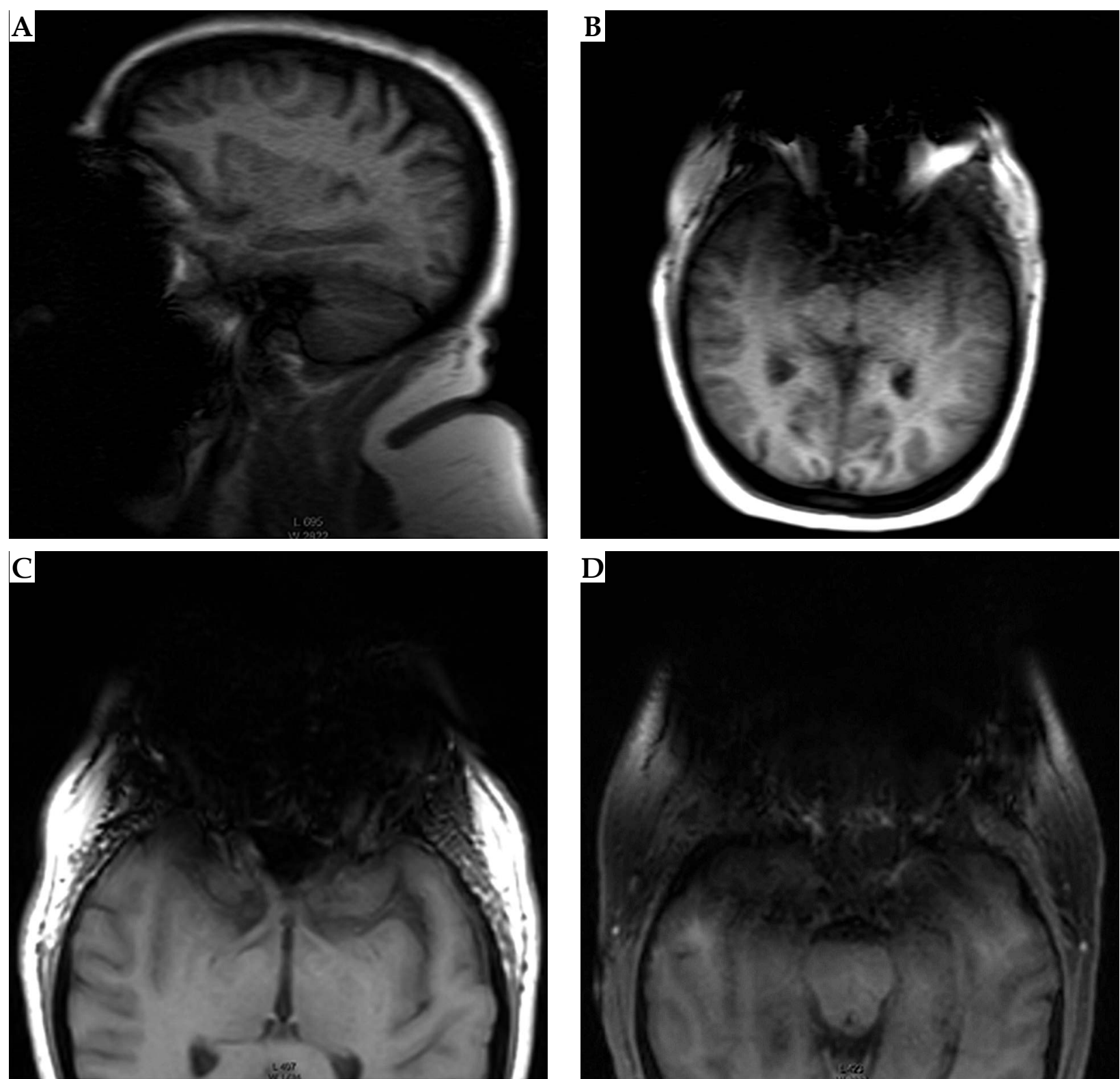

Fig. 1. Conventional T1W 2D scout (A, B) and 3D structural (C, D) magnetic resonance images (MRIs) showing signal loss in the anterior portion of the head from dental braces. A) Sagittal scout. B) Axial scout. C) Axial 3D T1W MRI of orbits in slice containing the tumor. D) Slice through maxillary sinuses $16 \mathrm{~mm}$ inferior of the tumor. Images were windowed to display the artifacts 

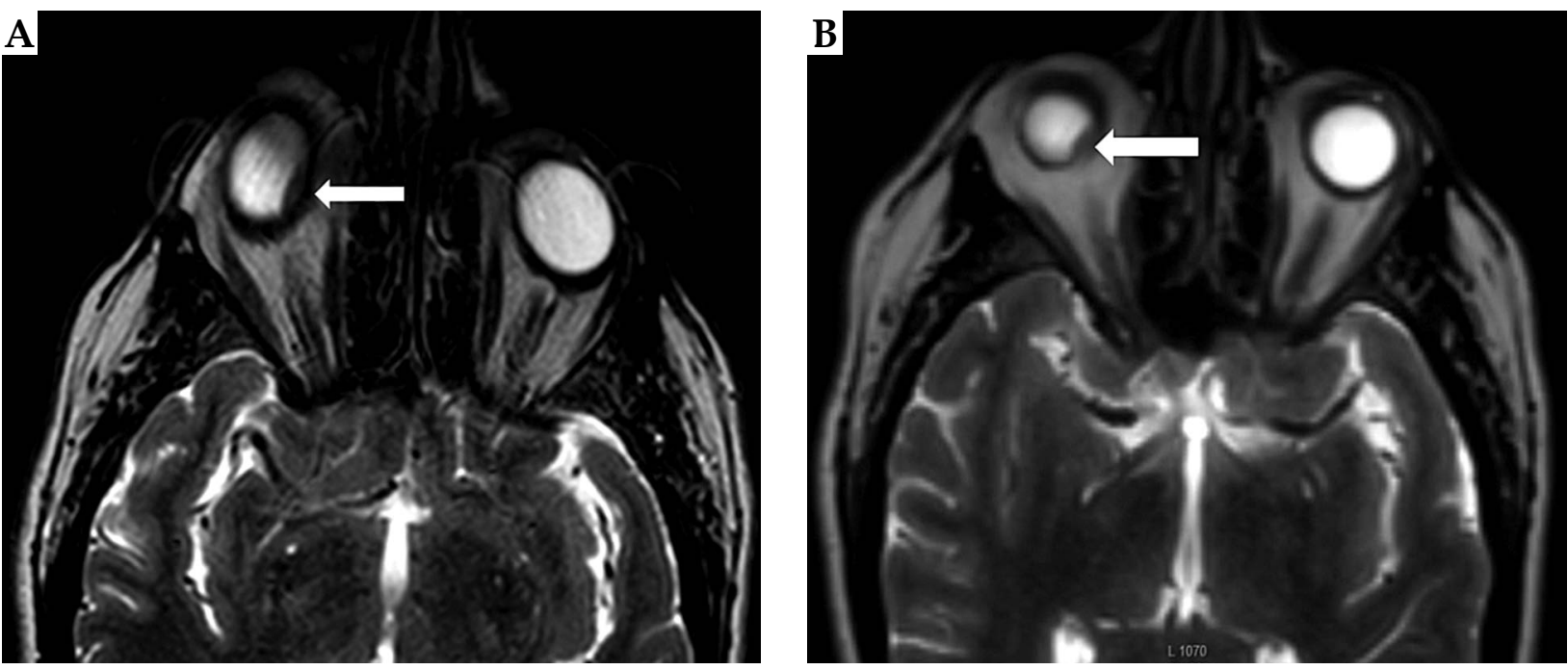

Fig. 2. A) Conventional axial T2W magnetic resonance image (MRI) of the orbits showing the geometric distortion associated with magnetic susceptibility and motion artifacts. B) Axial T2W with O-MAR. The tumor is indicated by the arrow. The conventional T2W images appear grainier than the O-MAR images due to the former's smaller reconstruction pixel size and slice thickness

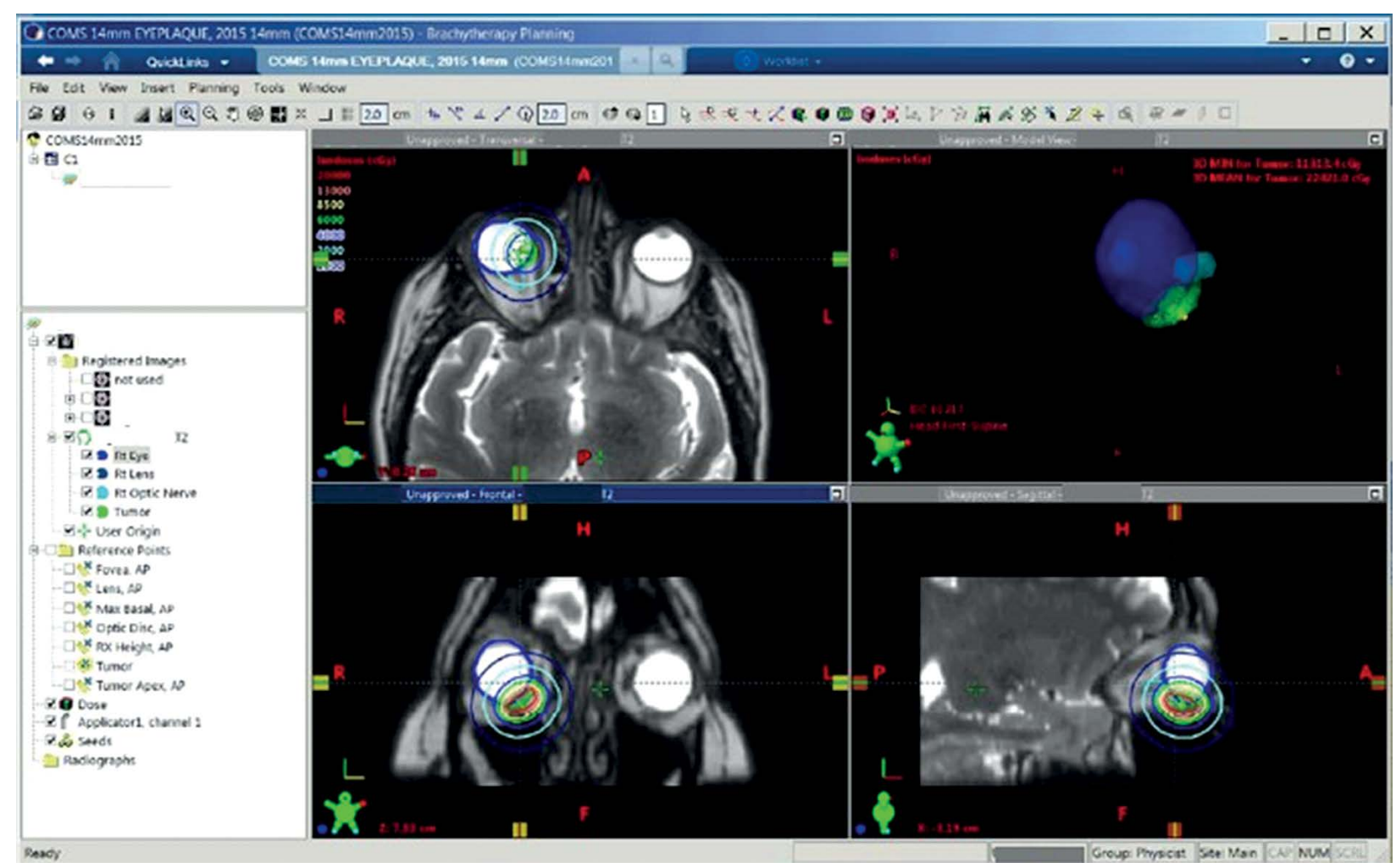

Fig. 3. Magnetic resonance imaging-based 3D treatment plan for patient's eye plaque brachytherapy using T2W O-MAR images

Table 2. Total dose based on 3D treatment plan and 96 hour exposure

\begin{tabular}{lcccc} 
Reference point & $\mathrm{x}[\mathrm{cm}]^{*}$ & $\mathrm{y}[\mathrm{cm}]^{*}$ & $\mathrm{z}[\mathrm{cm}]^{*}$ & Total dose $(\mathrm{cGy})^{*}$ \\
\hline RX height, AP & -3.43 & -0.03 & 8.21 & 8615.6 \\
\hline Tumor apex, AP & -3.38 & -0.11 & 8.19 & 10827.7 \\
\hline Max. basal, AP & -3.19 & -0.37 & 8.12 & 27580.3 \\
\hline Lens, AP & -3.90 & 0.89 & 9.03 & 1098.9 \\
\hline Fovea, AP & -3.69 & 0.32 & 7.37 & 2848.6 \\
\hline Optic disc, AP & -3.40 & 0.18 & 7.47 & 4979.9
\end{tabular}

*IEC 61217 coordinate system 
in MRI to be $7.9 \times 9.1 \times 4.1 \mathrm{~mm}$ with a maximal dimension of $9.4 \mathrm{~mm}$. The posterior tumor edge to the fovea and disk were 6 and $4 \mathrm{~mm}$, respectively. The prescription (RX) height was $4.3 \mathrm{~mm}$.

The patient received a COMS $14 \mathrm{~mm}$ plaque (unnotched) containing 13 seeds with $3.84 \mathrm{U} /$ seed $(3.02 \mathrm{mCi} /$ seed). The treatment time was 95 hours and 12 minutes.

\section{Discussion}

MRI metal artifact reduction was previously used to reduce the effects of metallic dental implants in MRI and PET/MRI [21,22]. This is the first published report of metal artifact reduction to suppress MRI artifacts resulting from dental braces. Unfortunately, we were unable to evaluate the T1W O-MAR sequence or try other pulse sequence settings due to the inability of the patient to remain in the MRI because of her claustrophobia.

Previously, there were few options for minimizing artifacts from dental braces. Increasing the receiver bandwidth (readout gradient amplitude) could significantly decrease the image distortion observed in the conventional T2W images near the orbits albeit at the cost of decreased signal-to-noise and contrast-to-noise ratios. However, increasing the receiver bandwidth on the conventional T1W MRIs would not have adequately corrected the artifacts and signal loss.

In this patient, the medium SEMAC setting was satisfactory for imaging the orbits in the presence of dental braces. Assuming no signal averaging and an equivalent number of slices, the medium SEMAC T2W MRIs required a roughly three times longer acquisition time than the conventional T2W MRIs. Hence, the acquisition time was a major disadvantage of the O-MAR method. However, MRI acquisitions of seven minutes are routine for imaging the whole brain (e.g., 3D T1W MRIs). In addition, accelerated O-MAR acquisitions were recently demonstrated using compressed sensing to significantly reduce the acquisition time [23].

The strong SEMAC setting (with 21 z-phase encodes) may be useful for more severe artifacts or imaging volumes that are closer to the source of the artifact. However, the strong setting increases the acquisition time by $\sim 60 \%$ relative to the medium SEMAC setting. Alternatively, O-MAR with the weak SEMAC setting may be useful for volumes of interest near metals that produce mild susceptibility artifacts e.g., the eye after implantation of the metal eye plaque.

Without the metal artifact reduction, the MRIs would not have been satisfactory to generate a $3 \mathrm{D}$ treatment plan. Instead, the treatment plan would have relied on either a 2D model based on fundoscopy and ultrasound imaging [24] or a 3D plan based on CT images. Therefore, the advantages of O-MAR in facilitating an MRI-based 3D treatment plan for this patient outweighed the disadvantage of increased acquisition time.

\section{Conclusions}

In conclusion, O-MAR was demonstrated to provide excellent reduction of susceptibility artifacts and geomet- ric distortion in T2W MRI of the orbits caused by dental braces. The MRIs with metal artifact reduction had sufficient quality to be used in the patient's brachytherapy treatment planning whereas the conventional MRIs were unusable. Therefore, temporary removal of the dental braces was avoided by using O-MAR.

\section{Disclosure}

Mo Kadbi is an employee of Philips Healthcare, which supplied the O-MAR pulse sequence used in this case report. There are no other conflicts of interest.

\section{References}

1. Knutsen S, Hafslund R, Monge OR et al. Dosimetric verification of a dedicated 3D treatment planning system for episcleral plaque therapy. Int J Radiat Oncol Biol Phys 2001; 51: 1159-1166.

2. Hawkins BS. Collaborative ocular melanoma study randomized trial of I-125 brachytherapy. Clin Trials 2011; 8: 661-673.

3. Finger PT, Zhou D, Kalach N et al. 103Pd versus 125I ophthalmic plaque brachytherapy: preoperative comparative radiation dosimetry for 319 uveal melanomas. J Radiat Oncol 2014; 3: 409-416.

4. Naseripour M, Jaberi R, Sedaghat A et al. Ruthenium-106 brachytherapy for thick uveal melanoma: reappraisal of apex and base dose radiation and dose rate. J Contemp Brachytherapy 2016; 8: 66-73.

5. Laskar S, Gurram L, Laskar SG et al. Superficial ocular malignancies treated with strontium-90 brachytherapy: long term outcomes. J Contemp Brachytherapy 2015; 7: 369-373.

6. Wisely CE, Hadziahmetovic M, Reem RE et al. Long-term visual acuity outcomes in patients with uveal melanoma treated with 125I episcleral OSU-Nag plaque brachytherapy. Brachytherapy 2016; 15: 12-22.

7. Gagne NL, Rivard MJ. Quantifying the dosimetric influences of radiation coverage and brachytherapy implant placement uncertainty on eye plaque size selection. Brachytherapy 2013; 12: 508-520.

8. Garcia-Ramirez JL, Esthappan J, Mansur D et al. Three-dimensional CT treatments pre-planning of I-125 COMS eye plaque implant brachytherapy (Abstract PO72). Brachytherapy 2008; 7: 162.

9. Zoberi JE, Garcia-Ramirez J, Hedrick S et al. MRI-based treatment planning and dose delivery verification for intraocular melanoma brachytherapy. Brachytherapy 2017; doi: 10.1016/ j.brachy.2017.07.011 [Epub ahead of print].

10. Tarlan B, Kiratlı H. Uveal melanoma: Current trends in diagnosis and management. Turk J Opthamol 2016; 46: 123-137.

11. Houdek PV, Schwade JG, Medina AJ et al. MR technique for localization and verification procedures in episcleral brachytherapy. Int J Radiat Oncol Biol Phys 1989; 17: 1111-1114.

12. Daftari I, Aghaian E, O'Brien JM et al. 3D MRI-based tumor delineation of ocular melanoma and its comparison with conventional techniques. Med Phys 2005; 32: 3355-3362.

13. Elison JM, Leggitt VL, Thomson M et al. Influence of common orthodontic appliances on the diagnostic quality of cranial magnetic resonance images. Am J Orthod Dentofacial Orthop 2008; 134: 563-572.

14. New PF, Rosen BR, Brady TJ et al. Potential hazards and artifacts of ferromagnetic and nonferromagnetic surgical and dental materials and devices in nuclear magnetic resonance imaging. Radiology 1983; 147: 139-148.

15. Poorsattar-Bejeh Mir A, Rahmati-Kamel M. Should the orthodontic brackets always be removed prior to magnetic 
resonance imaging (MRI)? J Oral Biol Craniofac Res 2016; 6: 142-152.

16. Cho ZH, Kim DJ, Kim YK. Total inhomogeneity correction including chemical shifts and susceptibility by view angle tilting. Med Phys 1988; 15: 7-11.

17. Lu W, Pauly KB, Gold GE et al. SEMAC: Slice encoding for metal artifact correction in MRI. Magn Reson Med 2009; 62: 66-76.

18. Rao YJ, Zoberi JE, Kadbi M et al. Metal artifact reduction in MRI-based cervical cancer intracavitary brachytherapy. Phys Med Biol 2017; 62: 3011-3024.

19. Nath R, Anderson LL, Luxton G et al. Dosimetry of interstitial brachytherapy sources: recommendations of the AAPM Radiation Therapy Committee Task Group No. 43. American Association of Physicists in Medicine. Med Phys 1995; 22: 209-234.

20. Chiu-Tsao ST, Astrahan MA, Finger PT et al. Dosimetry of (125)I and (103)Pd COMS eye plaques for intraocular tumors: report of Task Group 129 by the AAPM and ABS. Med Phys 2012; 39: 6161-6184.

21. Gunzinger JM, Delso G, Boss A et al. Metal artifact reduction in patients with dental implants using multispectral three-dimensional data acquisition for hybrid PET/MRI. EJNMMI Phys 2014; 1: 102.

22. Probst M, Richter V, Weitz J et al. Magnetic resonance imaging of the inferior alveolar nerve with special regard to metal artifact reduction. J Craniomaxillofac Surg 2017; 45: 558-569.

23. Fritz J, Ahlawat S, Demehri $S$ et al. Compressed Sensing SEMAC: 8-fold Accelerated High Resolution Metal Artifact Reduction MRI of Cobalt-Chromium Knee Arthroplasty Implants. Invest Radiol 2016; 51: 666-676.

24. Badiyan SN, Rao RC, Apicelli AJ et al. Outcomes of iodine-125 plaque brachytherapy for uveal melanoma with intraoperative ultrasonography and supplemental transpupillary thermotherapy. Int J Radiat Oncol Biol Phys 2014; 88: 801-805. 